\title{
A Tumbling Magnetic Microrobot System for Biomedical Applications
}

\author{
Elizabeth E. Niedert ${ }^{1,+}$, Chenghao Bi ${ }^{2,+}$, Georges Adam ${ }^{2}$ (D), Elly Lambert ${ }^{1}$, Luis Solorio ${ }^{1,3}{ }^{(D)}$, \\ Craig J. Goergen ${ }^{1,3}\left(\mathbb{D}\right.$ and David J. Cappelleri ${ }^{2, *} \mathbb{D}$ \\ 1 Weldon School of Biomedical Engineering, Purdue University, West Lafayette, IN 47907, USA; \\ eniedert@purdue.edu (E.E.N.); lamberte@purdue.edu (E.L.); lsolorio@purdue.edu (L.S.); \\ cgoergen@purdue.edu (C.J.G.) \\ 2 School of Mechanical Engineering, Purdue University, West Lafayette, IN 47907, USA; \\ bi10@purdue.edu (C.B.); adamg@purdue.edu (G.A.) \\ 3 Center for Cancer Research, Purdue University, West Lafayette, IN 47907, USA \\ * Correspondence: dcappell@purdue.edu \\ + These authors contributed equally to this work.
}

Received: 10 July 2020; Accepted: 15 September 2020; Published: 17 September 2020

\begin{abstract}
A microrobot system comprising an untethered tumbling magnetic microrobot, a two-degree-of-freedom rotating permanent magnet, and an ultrasound imaging system has been developed for in vitro and in vivo biomedical applications. The microrobot tumbles end-over-end in a net forward motion due to applied magnetic torque from the rotating magnet. By turning the rotational axis of the magnet, two-dimensional directional control is possible and the microrobot was steered along various trajectories, including a circular path and P-shaped path. The microrobot is capable of moving over the unstructured terrain within a murine colon in in vitro, in situ, and in vivo conditions, as well as a porcine colon in ex vivo conditions. High-frequency ultrasound imaging allows for real-time determination of the microrobot's position while it is optically occluded by animal tissue. When coated with a fluorescein payload, the microrobot was shown to release the majority of the payload over a 1-h time period in phosphate-buffered saline. Cytotoxicity tests demonstrated that the microrobot's constituent materials, SU-8 and polydimethylsiloxane (PDMS), did not show a statistically significant difference in toxicity to murine fibroblasts from the negative control, even when the materials were doped with magnetic neodymium microparticles. The microrobot system's capabilities make it promising for targeted drug delivery and other in vivo biomedical applications.
\end{abstract}

Keywords: magnetic microrobots; biomedical microrobots; drug delivery

\section{Introduction}

Recent advances in the design and fabrication of microrobots has made them increasingly viable for biomedical applications [1-3]. Due to their small size, microrobots have the potential to access many areas of the body with minimally invasive strategies. They can be wirelessly controlled and steered toward target locations within the body to perform a myriad of tasks. Compared to conventional surgical and drug administration techniques, the use of actively guided microrobots have promise to reduce patient trauma, lower the risk of side effects, and have higher drug retention rates.

Potential applications such as microsurgery [4], tumor imaging and ablation [5,6], tissue biopsies [7], targeted drug delivery [8-10], cell delivery [11,12], and gene silencing [13,14] have recently been explored, with demonstrations of microrobot viability in both in vitro and in vivo conditions. Polymer nanoplatforms have been shown to release chemicals to different stimuli such as presence of certain enzymes, $\mathrm{pH}$ changes, temperature differences, ultrasound, etc. [7]. Colloid micromotors with a 
cell membrane coating show biocompatibility and movement with outside triggers [15]. Microcapsules were triggered to open in live mice using ultrasound [16]. An acid-driven microrobot was used to press a drug payload directly against the stomach walls of live mice [17]. Tetherless microgrippers were shown to capture live fibroblast cell clusters in vitro [18] and perform in vivo biopsies of porcine bile ducts [19]. High speed, ultrasound-actuated microbullets were able to perform deep tissue penetration, deformation, and cleaving in vitro [4]. Localized motion and continuous fluid mixing from various micromotors led to significantly accelerated results in immunoassay recognition [20], toxin neutralization [21], and ion binding compared to similar static techniques [22]. Although these results are promising, the translation of microrobots from the laboratory setting to a clinical setting remains a daunting task.

A critical challenge for the use of microrobots in vivo is the difficulty of real-time spatial localization in the presence of visual occlusions. Microrobots are too small for on-board power or computation; they cannot broadcast or determine their location autonomously. Thus, external imaging tools are necessary for microrobot localization. Imaging methods employing visible light are not suitable for minimally invasive operations, where tissue blocks the line of sight. Alternative methods capable of penetrating tissue are therefore necessary. Such methods include optical fluorescence imaging [23-27], X-ray analysis [28,29], ultrasound imaging [30,31], and magnetic resonance imaging (MRI) [32,33]. Potential problems from these methods arise from poor spatial and temporal resolution [34], bulky equipment, and undesired interactions between microrobot imaging and actuation methods. Magnetic actuation is difficult to use simultaneously with MRI imaging due to phenomena such as field distortion caused by interactions between multiple magnetic field sources [32]. It might also be impractical to fit certain imaging and actuation systems within the confines of a clinical workspace. An imaging/actuation combination with high resolution, cross-compatibility, small footprint, and tissue penetration capabilities is necessary for the feasibility of actively guided, minimally invasive in vivo microrobots. High-frequency ultrasound imaging ( $>10 \mathrm{MHz}$ ) was combined with magnetic actuation to localize tumbling magnetic microrobots to investigate biomedical applications in our prior work [35].

In this paper, we significantly expound on this work investigating the overall tumbling microrobot system efficacy for various biomedical environments. Specifically, we used a novel two-degree-of-freedom rotating permanent magnet system as the source of the time-varying external magnetic field for wireless control and propulsion. The fabrication of two different microrobot material versions were investigated. Cytotoxicity tests confirmed that the microrobots' constituent materials were not statistically different in toxicity to murine fibroblasts from the negative control. The microrobots were observed and locomote in two-dimensions over an agarose block (in vitro), inside a porcine colon (ex vivo), inside a euthanized murine colon (in situ), and inside a live murine colon (in vivo). Additionally, the relationship between microrobot velocity vs. the viscosity of the surrounding medium was studied. Force measurements showed that the forces exerted by the moving microrobots are not large enough to puncture or damage internal tissues. Using an electrospraying process, the microrobots are functionalized with a fluorescein payload that diffused over an extended time span, indicating viability for drug delivery applications. In this paper, we highlight the development of a microrobot system consisting of high-frequency ultrasound imaging, magnetic actuation, and tumbling magnetic microrobots, ultimately showing promise for a variety of minimally invasive in vivo biomedical applications.

\section{Materials and Methods}

\subsection{Microrobot Motion Principle}

Due to differences between the alignment of the tumbling microrobot's magnetic polarity and that of the external actuating field, seen in Figure 1, a magnetic torque is exerted on the robot:

$$
\overrightarrow{T_{m}}=V_{m} \vec{M} \times \vec{B}
$$


Equation (1) describes the general working principle of this torque, where $V_{m}$ is the magnetic volume of the robot, $\vec{M}$ is the magnetization of the robot, and $\vec{B}$ is the external magnetic field strength. Under a time-varying rotating magnetic field, the torque causes the microrobot to tumble end-over-end, resulting in a net forward motion.

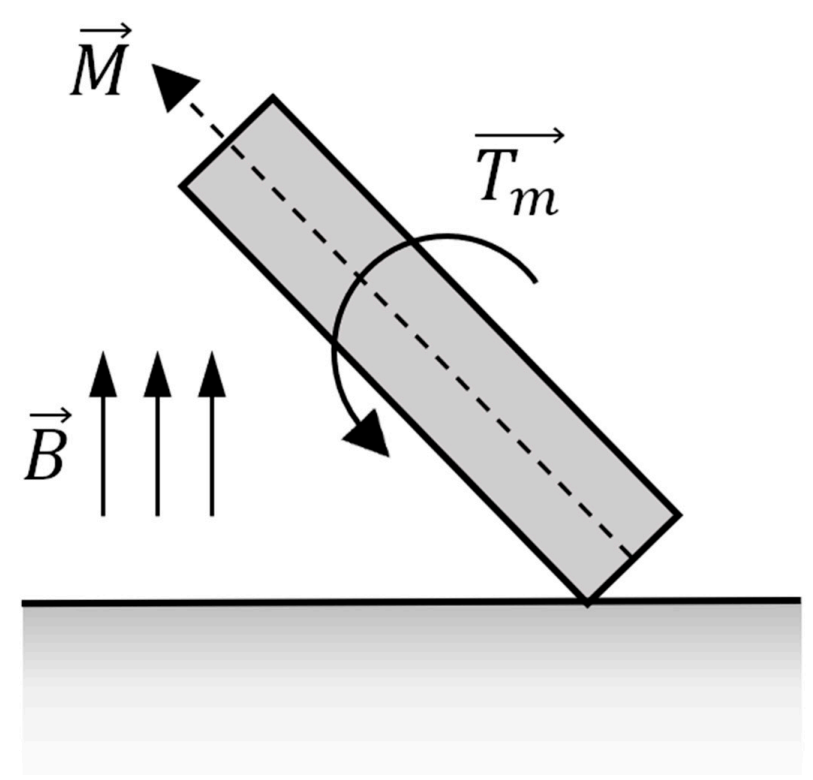

Figure 1. Diagram of magnetic alignments and resultant magnetic torque.

\subsection{Microrobot Fabrication Method}

Figure S1 summarizes the entire fabrication and magnetization procedure for the SU-8 microrobot variant. First, SU-8 50 photoresist is doped with NdFeB particles (Magnequench MQFT $5 \mu \mathrm{m}$, Neo Magnequench) at a concentration of $15 \mathrm{~g} / 50 \mathrm{~mL}$. The doped SU-8 is then spin-coated at $1000 \mathrm{rpm}$ for $60 \mathrm{~s}$ and undergoes a two-step soft-baking process of $10 \mathrm{~min}$ at $65^{\circ} \mathrm{C}$ and $30 \mathrm{~min}$ at $95^{\circ} \mathrm{C}$. These steps are used to obtain a thick layer of SU-8, approximately $120 \mu \mathrm{m}$, and to evaporate the excess solvent. Next, the wafer is exposed to UV light in a mask aligner (Suss MA6 Mask Aligner, SUSS MicroTec AG, Garching, Germany) using a mask corresponding to the geometry of the microrobot for $70 \mathrm{~s}$. A postexposure bake of $1 \mathrm{~min}$ at $65^{\circ} \mathrm{C}$ and $10 \mathrm{~min}$ at $95^{\circ} \mathrm{C}$ is then performed to selectively cross-link the exposed areas of the film. Lastly, the nonpolymerized SU-8 is removed with SU-8 developer (MicroChem) in a bath for $10 \mathrm{~min}$, and then, the wafer is cured in an oven at $160{ }^{\circ} \mathrm{C}$.

For PDMS microrobot variant, the fabrication procedure is slightly different: a mold for the microrobot is first created and then PDMS is added to complete the fabrication. First, KMPR 1000 (MicroChem), a negative epoxy photoresist, is patterned on the substrate by spin-coating it at $500 \mathrm{rpm}$ for $30 \mathrm{~s}$ and $1000 \mathrm{rpm}$ for $30 \mathrm{~s}$, followed by a soft-bake at $100{ }^{\circ} \mathrm{C}$ for $5 \mathrm{~min}$. Then, using a mask aligner (Suss MA6 Mask Aligner, Suss MicroTec AG) and a mask corresponding to the mold of the microrobot, a negative image of the microrobot shape is produced. A postexposure bake at $100{ }^{\circ} \mathrm{C}$ for $20 \mathrm{~min}$ followed by a bath in SU-8 developer completes the fabrication of the microrobot mold. Next, the PDMS elastomer is mixed with the curing agent at a ratio of 10:1, and then, the magnetic particles are mixed in with the same concentration as before. The doped PDMS is spread over the mold using a silicone spatula, removing the excess, and then cured at $50{ }^{\circ} \mathrm{C}$ for approximately one day. Lastly, the KMPR mold is removed by placing it in a PG remover bath, thus releasing the PDMS microrobot.

Once the microrobots are fabricated, they are manually removed from the wafer and the embedded magnetic particles are then aligned along the same direction through brief exposure to a uniform external magnetic field $9 \mathrm{~T}$ in strength. This magnetization step significantly improves the uniformity 
of the magnetic alignment and remanent magnetic strength of the particles, enhancing microrobot responsiveness under lower magnetic field strengths. The microrobot is secured in the desired orientation during the magnetization process on a quartz sample holder using Kapton tape (Dupont). The external field is generated using a PPMS DynaCool machine (Quantum Design), which is capable of applying uniform magnetic fields of up to $9 \mathrm{~T}$. The magnetization process allows for different magnetic polarity alignments irrespective of the microrobot's geometry or the physical orientation of the magnetic particles.

\subsection{Payload Coating}

Payload coating was completed utilizing electrospraying (Figures S2 and S3). Microrobots were coated with a solution consisting of a 50:50 ratio of dimethylformamide (DMF):chloroform, $1 \%$ poly(lactic-co-glycolic acid) (PLGA), and 1\% fluorescein, with fluorescein serving as a mock drug payload. Spraying was performed between 5.5 and $6.2 \mathrm{kV}$ for $1 \mathrm{~h}$ per side of the microrobots and dried for 5 days at room temperature in dark conditions.

\subsection{Force Measurement Method}

Figure $\mathrm{S} 4$ shows the system used to measure the force applied by the microrobot on the surface as it is moving. A three-degree-of-freedom micromanipulator (MP-225, Sutter Instruments, Novato, CA, USA) with a resolution of $1 \mu \mathrm{m}$ per step size is used to place a MEMS force sensor (FT-100, FemtoTools) close to the workspace so that the microrobot is able to hit it as it is rotating. The magnetic actuation system is used to move the microrobot along the workspace and aim it at the tip of the MEMS force sensor.

For both the static and dynamic tests, the microrobot was actuated on a rigid surface (glass slide) at the same relative distance from the MEMS force sensor. The variability in the results come from the fact that the microrobot does not always directly contact the sensor, as described earlier.

\subsection{In Vivo Locomotion Procedure}

For the in vivo tests, C57BL/6 male apolipoprotein $\mathrm{E}\left(a p o E^{-/-}\right)$knockout mice at 1 year of age were used. The animals were prepared for colon imaging by being fasted for $8-16 \mathrm{~h}$ beforehand [36]. Using isoflurane anesthesia, the mice were anesthetized and placed on the sample stage above the motorized magnet manipulator. The mice were secured with tape and a heat lamp was placed nearby to maintain normal body temperatures. Hair was removed from the lower abdomen and around the anus using a depilatory cream. The colon was flushed with $1 \mathrm{~mL}$ of ultrasound gel followed by about $1 \mathrm{~mL}$ of saline to rid the colon of remaining feces. An injection of atropine $(0.02 \mathrm{mg} / \mathrm{mL}$; \#A0132, Sigma-Aldrich), 100-150 $\mu \mathrm{L} \mathrm{SC,} \mathrm{was} \mathrm{given} \mathrm{to} \mathrm{halt} \mathrm{peristaltic} \mathrm{contractions} \mathrm{for} \mathrm{the} \mathrm{imaging} \mathrm{session} \mathrm{[36].}$ Then the colon was filled with saline, a microrobot was placed inside, and the colon was sealed off with a clothespin placed at the rectum. This saline containment caused the colon to be fully dilated, leaving enough space inside for the microrobot to move with minimal restriction. The Purdue Animal Care and Use Committee approved all animal experiments.

\subsection{Velocity Measurements}

Average velocities $(\bar{v})$ were calculated for each locomotion test condition using Equation (2):

$$
\bar{v}=\frac{\Delta x}{\Delta t}=\frac{x_{f}-x_{0}}{t_{f}-t_{0}}
$$

where $\Delta x$ represents the change in position, from the final position $\left(x_{f}\right)$ to the initial position $\left(x_{0}\right)$, and $\Delta t$ represents the change in time, from the final timepoint $\left(t_{f}\right)$ to the initial timepoint $\left(t_{0}\right)$. Position data were extracted from video recordings using MATLAB software (MathWorks). 


\section{Results}

\subsection{Microrobot Introduction}

The tumbling microrobot consists of an $800 \mu \mathrm{m} \times 400 \mu \mathrm{m} \times 100 \mu \mathrm{m}$ polymeric block that is doped with magnetic neodymium-iron-boron (NdFeB) microparticles (Figure $2 \mathrm{~A}$ ). A magnetic torque is applied on the microrobot due to differences in magnetic polarization between the microrobot and an external, time-varying magnetic field, resulting in a net forward tumbling motion. Two variants of the microrobot were fabricated: ones made out of rigid doped SU-8 photoresist and ones made out of elastomeric doped polydimethylsiloxane (PDMS) photoresist using standard photolithography techniques. An additional magnetization step was included to uniformly align and magnetically saturate the embedded particles by exposing the microrobots to a uniform $9 \mathrm{~T}$ magnetic field. The orientation of the microrobots under this external magnetic field determines their resultant tumbling behavior. Alignment along the length of the microrobot results in a lengthwise tumbling motion while alignment along the width of the robot results in a sideways tumbling motion (Figure 2B). Under the same magnetic field, lengthwise tumbling microrobots exhibit higher translational velocity than their sideways tumbling counterparts and also require more torque to rotate [37]. Lengthwise and sideways tumbling variants were fabricated for both the SU-8 and PDMS microrobots. Two circular cutouts, $100 \mu \mathrm{m}$ in diameter, allowed for additional surface area and empty volume to store payload substances (Figure 2C). The tumbling microrobots are capable of climbing inclines up to $60^{\circ}$ in fluid environments, moving over complex, unstructured terrain [37]. Demonstrated here, the microrobots are steered under open loop control to achieve desired trajectories (Figure 2D,E).

A
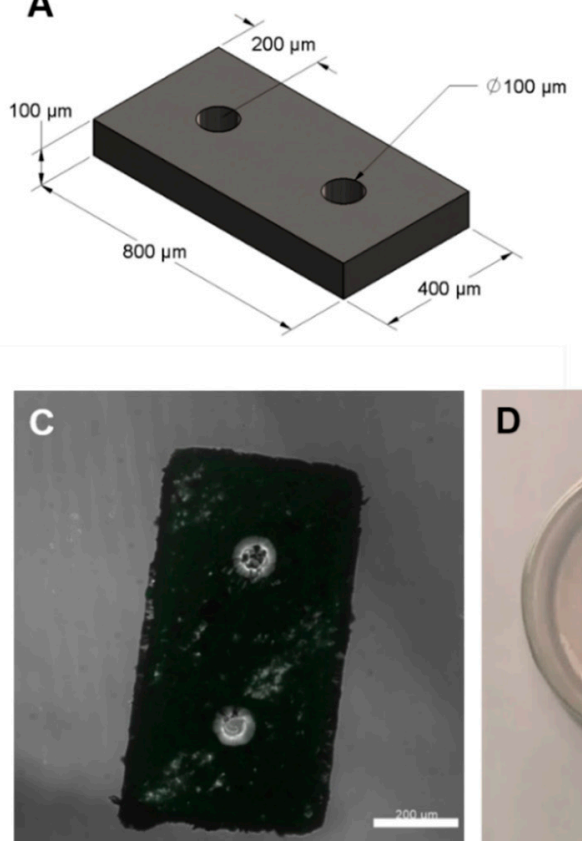

B
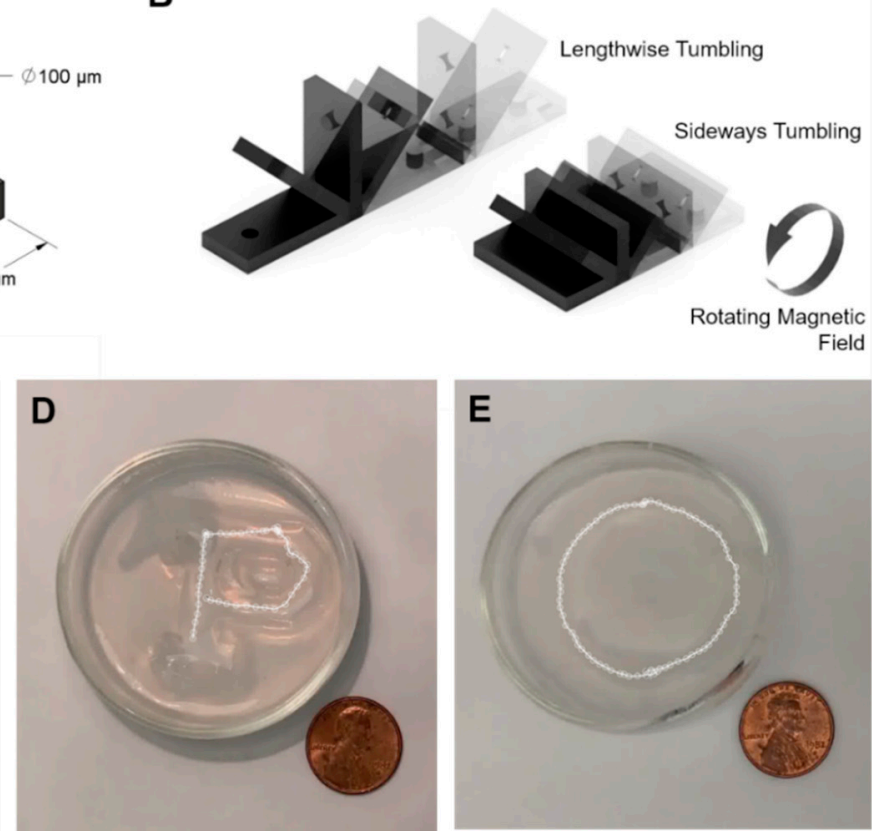

Figure 2. Tumbling magnetic microrobot overview. (A) Microrobot schematic with major dimensions. (B) No-slip lengthwise and sideways tumbling motions under rotating magnetic field. The sideways tumbling microrobot variant travels half the distance of the lengthwise microrobot variant under one complete rotation cycle. (C) Confocal microscope image of fabricated SU-8 microrobot. Scale bar is $200 \mu \mathrm{m}$. (D) P-shape and (E) circular shape trajectory of a lengthwise tumbling SU-8 microrobot moving in water over an indented agarose block. US penny for scale. 


\subsection{Cytotoxicity}

Prior to in vivo tests, the short-term cytotoxicity of SU-8 and PDMS were assessed. First, NIH3T3 murine fibroblasts were seeded in direct contact with the SU-8 materials, both in its doped and pure forms, and studied over the course of 3 days, with the initial measurements taken $12 \mathrm{~h}$ after initial seeding. NIH3T3 fibroblasts were also seeded on negative and positive controls consisting of tissue culture polystyrene and cells cultured in 70\% ethanol, respectively. Cell proliferation was examined using fluorescence microscopy (BioTek Cytation 5 Cell Imaging Multi-Mode Reader). Figure 3A indicates cell proliferation on the doped SU-8 material, suggesting that the cells do not exhibit signs of short-term toxicity. Initial seeding of the cells onto the polymer material may have been limited as seen in the slight decrease in cell expression in day 1 for the SU-8 material. However, an increase in living cells on days 3 and 5 indicate that cells were proliferating on the material. As expected, the negative control experienced cell proliferation while the positive control had no living cells after 3 days.
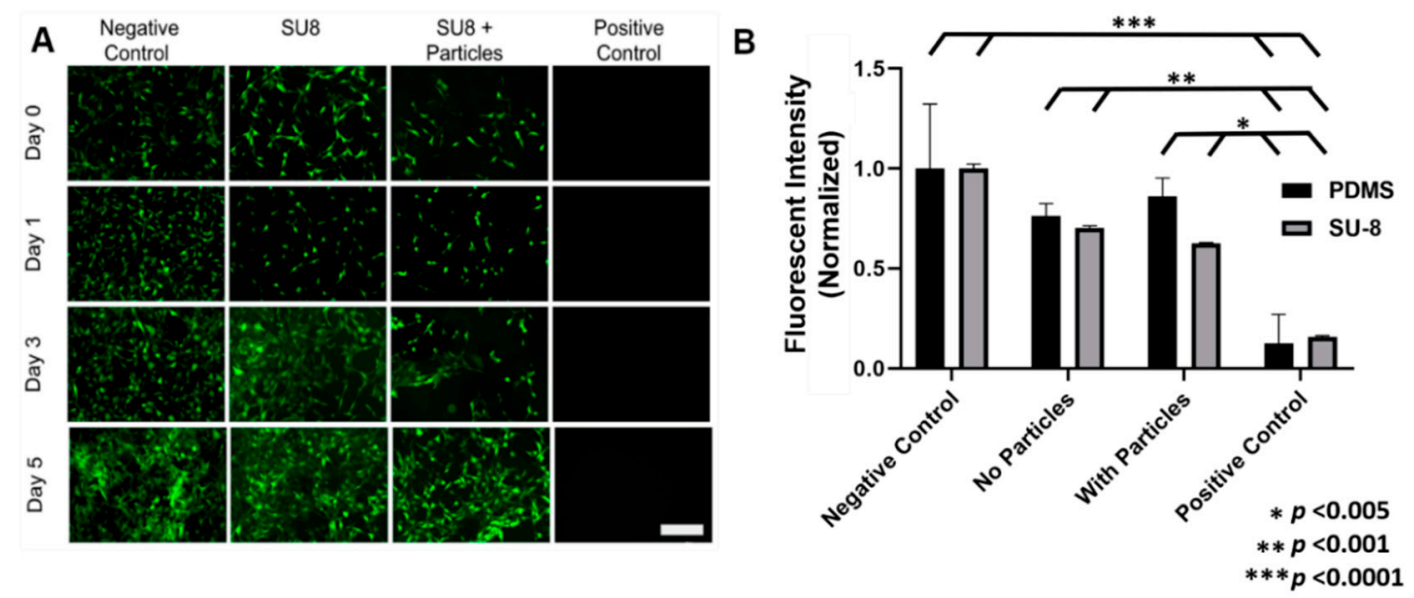

Figure 3. Cell viability on microrobot materials. (A) Fluorescent images taken of cell proliferation for four different test cases. Green fluorescent cells indicate living cells that have adhered to the well plate and are viable. Scale bar is $200 \mu \mathrm{m}$. (B) Cell viability was quantified utilizing a resazurin assay, normalized to the negative control (cell media) of the respective trials (SU-8 or PDMS). Significant differences were found between the negative control and positive control (*** $p<0.0001$ ), no particles and positive control (** $p<0.001)$, as well as with particles and positive control $\left({ }^{*} p<0.005\right)$ for both groups (SU-8 and PDMS).

The cytotoxicity assessment of PDMS followed a similar protocol as that of the SU-8. However, cells were seeded in a 24-well plate for $24 \mathrm{~h}$ before being exposed to pure PDMS and doped PDMS (Figure S5). After 3 days, cells still proliferated on both materials as well as the negative control. The positive control, again as expected, had no living cells after 3 days. Figure 3B shows cell viability, as a measure of normalized fluorescent intensity, of the different materials after 3 days, quantified using a resazurin assay. The cells were exposed to resazurin (Thermo Fisher, Waltham, MA, USA) for $2 \mathrm{~h}$ and absorbance was read to determine the metabolic capacity of the cells and quantify viability of each material (BioTek Cytation 5 Cell Imaging Multi-Mode Reader). Neither SU-8, PDMS, or their doped variants elicited a toxic response. Following ISO 10993-5, PDMS, PDMS + particles, and SU-8 did not elicit a cytotoxic response (a reduction of metabolic activity of less than $30 \%$ ). However, the SU-8 + particles had a reduced metabolic activity of 38\% prompting future investigation into the effect of the magnetic particles. Though the normalized cell viability percentages of SU-8 and PDMS were less than that of the negative control, their percentages were still well above that of the positive control, indicating nontoxicity for short-term in vivo applications. Although the neodymium particles were well-encapsulated by the photopolymers, the doped substances should still be removed from the body after microrobot operation to avoid potential heavy metal toxicity. 


\subsection{Locomotion Tests}

Real-time videos of the microrobots were acquired using a high-frequency ultrasound system (Vevo 3100, FUJIFILM VisualSonics, Toronto, ON, Canada) with the B-mode imaging setting. A linear array ultrasound probe (MX700) with a frequency range of 30-70 MHz and a central frequency of $50 \mathrm{MHz}$ was used for ultrasound imaging. With this transducer probe, the depth or axial resolution is limited to $30 \mu \mathrm{m}$. A cylindrical NdFeB permanent magnet $2.54 \mathrm{~cm}\left(1^{\prime \prime}\right)$ in diameter and $2.22 \mathrm{~cm}\left(0.875^{\prime \prime}\right)$ in height (Cyl1875, SuperMagnetMan) was rotated at set frequencies of $0.5,1.0$, and $1.5 \mathrm{~Hz}$ underneath the sample using a two-degree-of-freedom motorized magnet holder, applying magnetic torque on nearby magnetized objects. The location of the microrobot during locomotion is roughly $3.81 \mathrm{~cm}$ (1.5") above the magnet. Based on an analytical model of magnets with cylindrical symmetry [38], the magnetic flux density at the location of the microrobot is estimated to be $21.4 \mathrm{mT}$, although this value can fluctuate depending on the orientation of the magnet. Numerical simulations (COMSOL Multiphysics) of the magnetic field distribution estimate that the magnetic flux density due to the permanent magnet ranges from 12.5 to $19.4 \mathrm{mT}$ (Table S1). Continuous, reversible tumbling motion in a $180^{\circ}$ arc is possible, allowing the microrobot to be manipulated to any location on the planar sample space. Figure 4 illustrates the test setup and the degrees of freedom of the motorized permanent magnet manipulator. Major dimensions and components are detailed in Figure S6. The locomotion tests were conducted in ex vivo, in vitro, in situ dissected, in situ intact, and in vivo conditions to quantify microrobot performance in various biological settings.

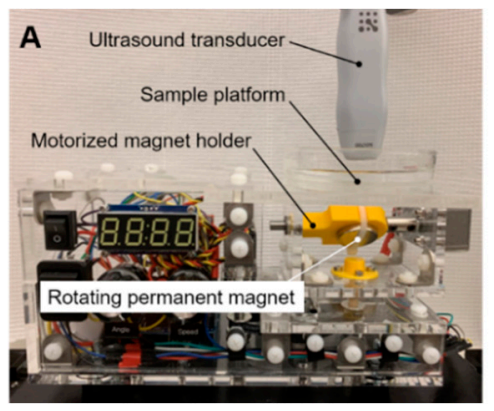

B

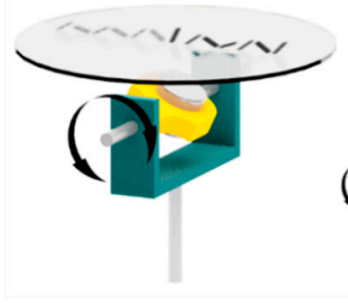

C

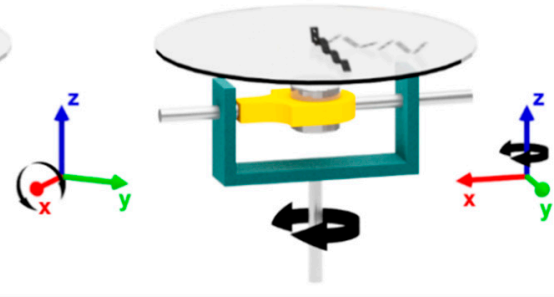

Figure 4. Locomotion test setup. (A) Schematic of setup. (B) Continuous, reversible rotation along the $x$-axis allows for forward and reverse tumbling motion. Coordinate triad corresponds to orientation of motorized magnet holder, represented by a grey U-bracket. (C) Reversible rotation along the $z$-axis with $180^{\circ}$ range allows for steering of the tumbling motion. Combining these two degrees of freedom allows the microrobot to be manipulated to any location on the planar sample platform.

\subsubsection{Ex Vivo Locomotion}

The translational velocities of four microrobot variants within a dissected porcine colon (ex vivo) were compared. These variants were the PDMS and SU- 8 microrobots that tumbled either lengthwise or sideways, and are hereafter referred to as PDMS lengthwise, PDMS sideways, SU-8 lengthwise, and SU-8 sideways. After one end of the colon was tied off, it was filled with water and a single microrobot was placed inside (Figure S7). The other end of the colon was subsequently sealed off with hemostats. The permanent magnet was rotated beneath the colon to induce tumbling motion and the visually occluded microrobot was then imaged with the ultrasound system.

All tested microrobots were able to move laterally across the colon at magnet rotation frequencies of $0.5,1.0$, and $1.5 \mathrm{~Hz}$. Figure 5A,B shows the SU-8 lengthwise microrobot moving under a rotation frequency of $1.0 \mathrm{~Hz}$ in the ex vivo porcine conditions. More than one microrobot can also move and be imaged within the colon at a time (Movie S1). Increased magnet rotation frequency resulted in an increase in the translational velocity of the microrobots in a roughly linear relationship (Figure S8). Table 1 lists the average velocities of the four microrobot variants across six trials for each rotation frequency. Trials were further organized based on the direction of the tumbling motion (forwards or 
backwards) due to its impact on the resulting microrobot velocity between each trial. Additionally, the previous microrobot is replaced with another one with the same design and a new starting location is used for each trial. A two-way ANOVA and the post hoc Tukey's test were run on the data and showed significance between materials, PDMS vs. SU-8, as well as between the tumbling orientation, lengthwise vs. sideways $[39,40]$. These tests were ran using GraphPad Prism v. 8.1.0 (GraphPad Software). The lengthwise tumbling microrobot variants were found to be faster than the sideways tumbling variants, as expected, and the PDMS microrobots were found to be slower than their SU-8 counterparts. Due to the higher average translation speeds observed for the SU-8 lengthwise microrobots compared to the other microrobot variants, these microrobots were used for all subsequent testing.
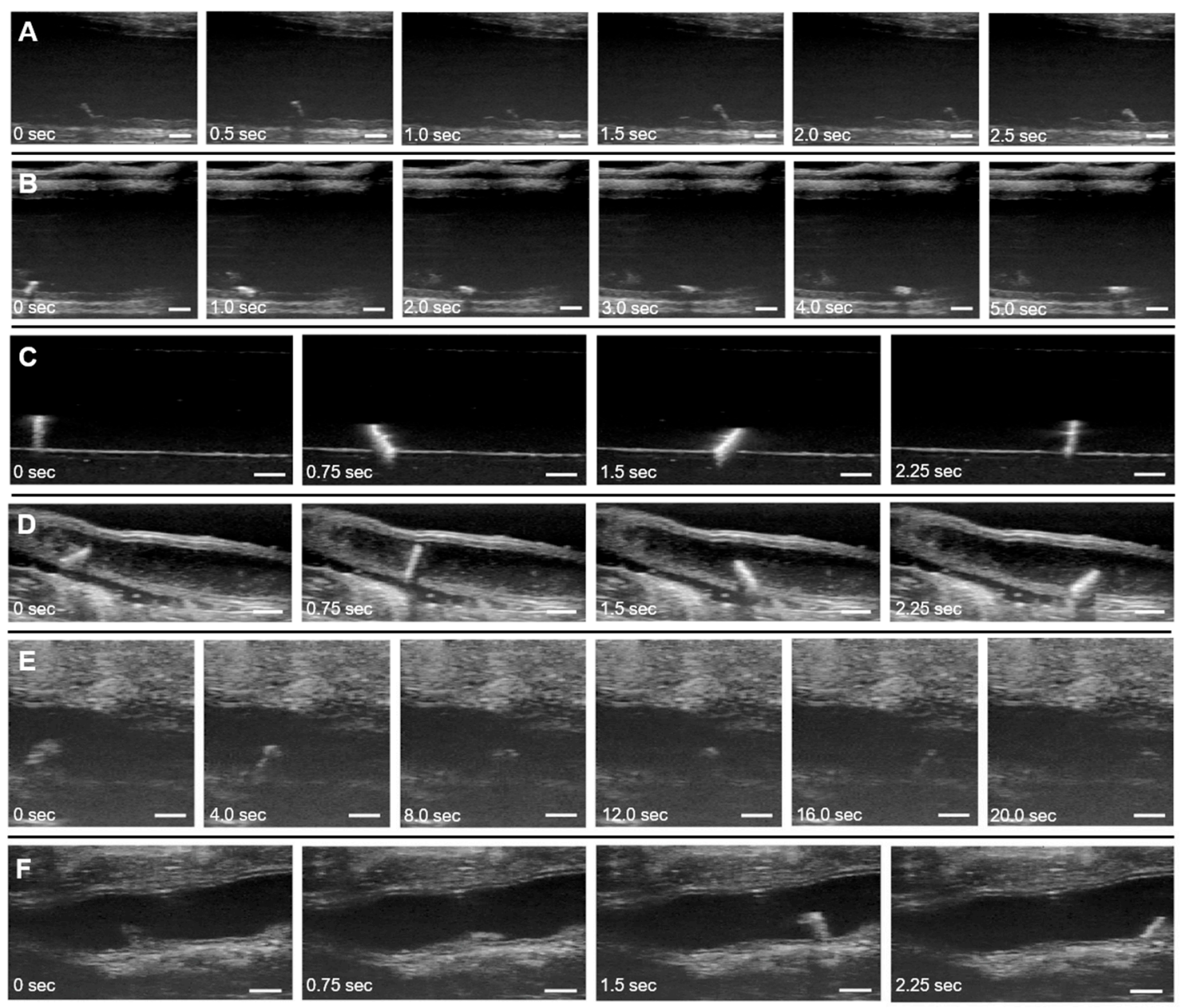

Figure 5. Real-time ultrasound B-Mode images of microrobots moving in ex vivo, in vitro, in situ, and in vivo conditions. Ultrasound images were analyzed using MATLAB (MathWorks, Natick, MA, USA) to incorporate a timestamp and larger scale bar while slowing down the frames to use for manual measurements. (A) SU-8 lengthwise microrobot moving in porcine colon in water (ex vivo). (B) SU-8 sideways microrobot moving in porcine colon in water (ex vivo). (C) SU-8 lengthwise microrobot moving in $1 \%$ agarose tunnel in water (in vitro). (D) SU-8 lengthwise microrobot moving in saline solution inside murine colon with tissue anterior to the colon removed (in situ dissected). (E) SU-8 lengthwise microrobot moving in intact colon of euthanized mouse in $1 \%$ Tylose solution (in situ intact). (F) SU-8 lengthwise microrobot moving in colon of live mouse in saline solution (in vivo). A magnet rotation frequency of $1 \mathrm{~Hz}$ was used in all of the images. Scale bars are $1 \mathrm{~mm}$. (Movie S1 shows microrobot locomotion in porcine colon tests; Movie S2 shows microrobot locomotion in agarose and murine colon tests.). 
Table 1. Microrobot velocities in ex vivo conditions.

\begin{tabular}{|c|c|c|c|c|c|c|c|c|}
\hline \multirow{3}{*}{ Frequency $(\mathrm{Hz})$} & \multirow{3}{*}{ Microrobot Type } & \multicolumn{6}{|c|}{ Trial } & \multirow{3}{*}{$\begin{array}{c}\text { Average } \\
\text { Velocity }(\mathrm{mm} / \mathrm{s})\end{array}$} \\
\hline & & \multicolumn{3}{|c|}{ Forwards Direction } & \multicolumn{3}{|c|}{ Reverse Direction } & \\
\hline & & 1 & 2 & 3 & 4 & 5 & 6 & \\
\hline \multirow{4}{*}{0.5} & PDMS lengthwise & 0.71 & 0.73 & 0.70 & 0.82 & 0.83 & 0.80 & $0.77 \pm 0.06$ \\
\hline & PDMS sideways & 0.49 & 0.50 & 0.51 & 0.24 & 0.24 & 0.24 & $0.37 \pm 0.14$ \\
\hline & SU-8 lengthwise & 0.74 & 0.63 & 0.74 & 1.18 & 1.00 & 1.08 & $0.89 \pm 0.22$ \\
\hline & SU-8 sideways & 0.83 & 0.84 & 0.88 & 0.54 & 0.79 & 0.62 & $0.75 \pm 0.14$ \\
\hline \multirow{4}{*}{1.0} & PDMS lengthwise & 1.53 & 1.48 & 1.56 & 1.69 & 1.73 & 1.69 & $1.61 \pm 0.10$ \\
\hline & PDMS sideways & 0.97 & 1.02 & 0.94 & 0.45 & 0.45 & 0.41 & $0.71 \pm 0.30$ \\
\hline & SU-8 lengthwise & 1.91 & 1.90 & 1.85 & 2.37 & 2.40 & 2.26 & $2.12 \pm 0.25$ \\
\hline & SU-8 sideways & 1.77 & 1.54 & 1.75 & 1.27 & 1.09 & 1.43 & $1.48 \pm 0.27$ \\
\hline \multirow{4}{*}{1.5} & PDMS lengthwise & 1.79 & 1.98 & 2.11 & 2.41 & 2.04 & 2.25 & $2.09 \pm 0.22$ \\
\hline & PDMS sideways & 1.49 & 1.45 & 1.21 & 0.75 & 0.68 & 0.64 & $1.04 \pm 0.39$ \\
\hline & SU-8 lengthwise & 2.94 & 2.52 & 2.60 & 3.57 & 3.57 & 3.67 & $3.14 \pm 0.52$ \\
\hline & SU-8 sideways & 2.48 & 2.07 & 2.33 & 1.67 & 1.74 & 1.91 & $2.03 \pm 0.32$ \\
\hline
\end{tabular}

\subsubsection{In Vitro Locomotion}

Figure 5C shows the microrobot traveling through a water-filled agarose tunnel. The $3.125 \mathrm{~mm}$ diameter tunnel was carved out of a $1 \%$ agarose (Thermo Fisher, Waltham, MA, USA) gel block, and the entire block was submersed in water over a glass dish, outside of any living organism (in vitro). Due to the uniformity of the agarose material and lack of complex tissues in this environment, the resultant ultrasound images showed the strong contrast between the microrobot and its surrounding environment.

\subsubsection{In Situ Dissected Locomotion}

In situ dissected tests were performed with the microrobot moving inside a colon from a euthanized C57BL/6 female apolipoprotein $\mathrm{E}\left(\mathrm{apoE}^{-/-}\right)$knockout mouse at 12 weeks of age. The tissue anterior to the colon was removed and a microrobot was then placed inside the colon through the anus (Figure S9). The colon was filled retrograde with saline ( $0.9 \%$ sodium chloride, Hanna Pharmaceuticals) and long-axis ultrasound images of the mid and distal regions were acquired [36]. The colon tissue was sutured on both ends to contain the saline and ensure the colon walls would not collapse on the microrobots inside, which would restrict motion. Figure 5D shows images from one of these experiments.

\subsubsection{In Situ Intact Locomotion}

For the in situ intact test case, the colon of a euthanized knockout mouse was left intact and a microrobot was again inserted into the anus of the mouse. The colon was filled with a $1 \%$ Tylose solution (HS 100,000 YP2, Shin-Etsu, Tokyo, Japan) instead of saline or water (Figure S10). This solution was much more viscous than the latter fluids, which allowed it to support the shape of the colon without the need of other constructs, such as sutures, to prevent the walls from collapsing and limiting microrobot motion (Figure 5E). The microrobot could not rotate in solutions even more viscous than $1 \%$ Tylose, such as standard ultrasound gel.

\subsubsection{In Vivo Locomotion}

For the in vivo test case, the murine preparation procedure for colon imaging used by Freeling et al. was followed and is further detailed in the experimental section [36]. The colon was filled with saline instead of $1 \%$ Tylose and the fluid was contained inside the colon by placing a clothespin on the rectum. An atropine injection was also used to halt peristaltic contractions of the colon during the 
test. These steps resulted in a test environment more favorable for imaging and microrobot movement, with a less viscous medium and fewer time-varying disturbances. Figure $5 \mathrm{~F}$ shows the microrobot locomotion for one of the in vivo tests.

The microrobot velocities in the in vitro, in situ, and in vivo conditions are recorded in Table 2. The magnet rotation frequency was kept at $1 \mathrm{~Hz}$ for all of these test conditions. Average velocities varied between the different conditions, reaching the highest magnitude in the aqueous in vitro tests and the lowest magnitude in the in situ intact tests in $1 \%$ Tylose. These differences are primarily due to the differing solution viscosities and terrains in each test environment. Table 3 shows the different viscosities that the SU-8 lengthwise microrobots were tested in with the microrobots being unable to move in ultrasound gel but having movement in other less viscous solutions. The $1 \%$ Tylose solution was much more viscous than the other aqueous solutions used. Although the viscosity of water is about $0.89 \mathrm{mPa}$, the viscosity of the $1 \%$ Tylose solution used was $4500 \mathrm{mPa}$ [41]. This increased viscosity led to more viscous drag, reducing the microrobot's velocity in the $1 \%$ Tylose solution to about a tenth of velocity exhibited in the aqueous conditions. The higher density of the $1 \%$ Tylose solution also led to increased buoyancy forces compared to the aqueous conditions, reducing traction between the microrobots and the substrate and causing them to slip during the tumbling motion. Additional differences in terrain heterogeneity, friction, and geometry, among other factors, led to varying results between test cases. Variation in velocity between trials was greater, e.g., in the in situ tests than in the in vitro tests. The homogeneous, flat surface of the in vitro tests allowed for more consistent motion between trials, while the complex, unstructured terrain of the organic environments in the in situ tests introduced more variation in microrobot velocities. Overall, the microrobots still maintained their ability to perform tumbling motion through in vitro, in situ, and in vivo conditions with repeatable, consistent speed in each test case.

Table 2. Microrobot velocities in in vitro, in situ, and in vivo conditions.

\begin{tabular}{ccccc}
\hline Test Condition & Water In Vitro & $\begin{array}{c}\text { Saline In Situ } \\
\text { Dissected }\end{array}$ & $\begin{array}{c}\text { 1\% Tylose } \\
\text { In Situ Intact }\end{array}$ & Saline In Vivo \\
\hline Trial 1 (mm/s) & 2.23 & 1.96 & 0.19 & 2.12 \\
Trial 2 (mm/s) & 2.21 & 1.89 & 0.19 & 2.03 \\
Trial 3 (mm/s) & 2.23 & 1.87 & 0.25 & 2.06 \\
Average velocity $(\mathrm{mm} / \mathrm{s})$ & $2.23 \pm 0.01$ & $1.91 \pm 0.05$ & $0.21 \pm 0.04$ & $2.07 \pm 0.05$ \\
\hline
\end{tabular}

Table 3. Velocities of the SU-8 microrobot under $1 \mathrm{~Hz}$ frequency of the magnet for a variety of solutions at various viscosities. Conditions were as follows: benchtop experiment for ultrasound gel, in situ intact murine colon for $1 \%$ Tylose solution, in vivo murine colon for saline, and ex vivo porcine colon for water.

\begin{tabular}{cccc}
\hline Solution & Velocity $(\mathbf{m m} / \mathbf{s})$ & Standard Deviation & Viscosity (mPa s) \\
\hline Ultrasound gel & 0 & 0 & $150,000[42]$ \\
1\% Tylose solution & 0.21 & 0.04 & $4500[41]$ \\
Saline & 2.07 & 0.05 & $1.092[43]$ \\
Water & 2.12 & 0.25 & 0.890 \\
\hline
\end{tabular}

\subsection{Payload Coating and Diffusion}

A payload coating process was performed on the microrobots and examined the payload's diffusion over time to investigate the potential of functionalizing the microrobots for drug delivery applications. Coating of the microrobots was completed via electrospraying them with a solution consisting of dimethylformamide (DMF), chloroform, poly(lactic-co-glycolic acid) (PLGA), and fluorescein. The drug diffusivity value is constant based on the polymer release of the mock drug payload. The circular cutouts allow for an increased surface area for the polymer to be coated on. This provides for an increased 
polymer and drug loading of about 3.50\%, however, the rate of diffusion would remain the same. The fluorescing microrobot in Figure 6A indicated a successful payload application on microrobots.
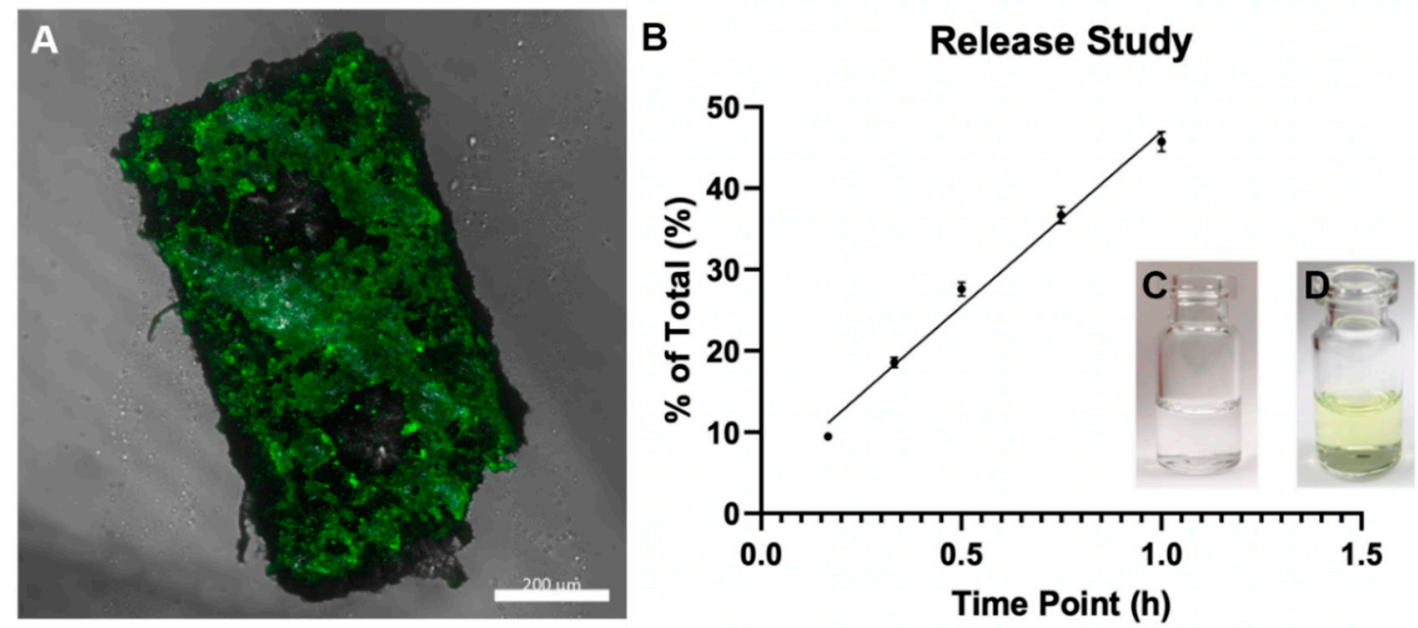

Figure 6. Payload diffusion results. (A) Confocal microscope image of fluorescing SU-8 microrobot after being coated. Scale bar is $200 \mu \mathrm{m}$. (B) Cumulative mass data of diffusion study for $60 \mathrm{~min}$ (C) Microrobot initially placed in glass vial in PBS solution. (D) Microrobot in vial and PBS $24 \mathrm{~h}$ later. Green solution is fluorescein released from PLGA coating.

Afterwards, the diffusion characteristics of the fluorescent payload were quantified. The coated microrobots were placed into $0.5 \mathrm{~mL}$ of phosphate buffered saline (PBS) in a $2 \mathrm{~mL}$ serum vial. These were kept at $37^{\circ} \mathrm{C}$ on a shaker at $100 \mathrm{rpm}$. Samples were taken from the bath-side solution at 10, 20, 30, 45, and $60 \mathrm{~min}$ after initial coating. The bath solution was replaced with fresh PBS at all sampling time points to maintain sink conditions. After $60 \mathrm{~min}$, the coated microrobots were dissolved in $\mathrm{NaOH}$ to determine any residual drug mass. The fluorescence of each sample was quantified afterwards using a Cytation 5 Cell Imaging Multi-Mode Reader (BioTek Instruments, Winooski, VT, USA). The samples were read at an excitation wavelength of $485 \mathrm{~nm}$ and emission wavelength of $525 \mathrm{~nm}$. The results shown in Figure 6B were obtained by comparing experimental measurements against a standard curve of absorbance values, which itself was generated by making solutions with known fluorescence concentrations. The experiment was run in triplicate to reduce the possibility of experimental bias or random error. Approximately 30\% of the payload releases from the microrobot in the first 30 min of diffusion and approximately half of the payload releases from the microrobot within the first hour. Given the microrobot's average in vivo speed of $2.07 \pm 0.05 \mathrm{~mm} / \mathrm{s}$ (Table 2), it has a theoretical travel range well over $1 \mathrm{~m}$ under no slip conditions before the majority of the payload diffuses from its body.

\subsection{Force Testing}

As the microrobots are intended to traverse inside and/or over a variety of biological tissues, it is important that the motion of robots does not damage these tissues. Actuation force tests of individual microrobots were conducted to quantify the amount of force they exert as they tumble over a surface. The theoretical maximum force was first calculated after assuming uniform magnetization of the microrobots and a field magnetic flux density of $21.4 \mathrm{mT}$. The resulting theoretical maximum force is approximately $43.6 \mu \mathrm{N}$.

Force measurement was conducted in a static test case where the individual microrobot started from a rest position (performing a half rotation and then recording the force) and a dynamic test case where the microrobot was already in motion (performing several rotations and then recording the force). Additionally, for the dynamic tests, magnet rotation frequencies were alternated between 1.0 and $1.5 \mathrm{~Hz}$ to explore any potential effects from a difference in speed. Results show that the forces remained around the same range for all cases (from 2 to $10 \mu \mathrm{N}$ ) with a few force spikes getting over 
$30 \mu \mathrm{N}$ (Table 4). Since the microrobot was manually aligned and subject to unpredictable surface forces, the microrobot did not always directly hit the force sensor resulting in the variance in the forces measured. Deviations from the ideal, straight-line contact caused variations in the resulting forces. Figure 7 outlines these deviations, showing the ideal contact position that results in the maximum force reading in Figure $7 \mathrm{~A}$ and also the possible misalignment that contributes to the large variance in force results in Figure 7B-D. The puncture force for pig (liver and skin) and other animal tissues ranges from approximately 0.2 to $2 \mathrm{~N}$ [44], a force four orders of magnitude greater than the maximum force the microrobot is capable of applying. Thus, it can be concluded that the tumbling motion of the microrobots does not run the risk of tissue damage or puncture.
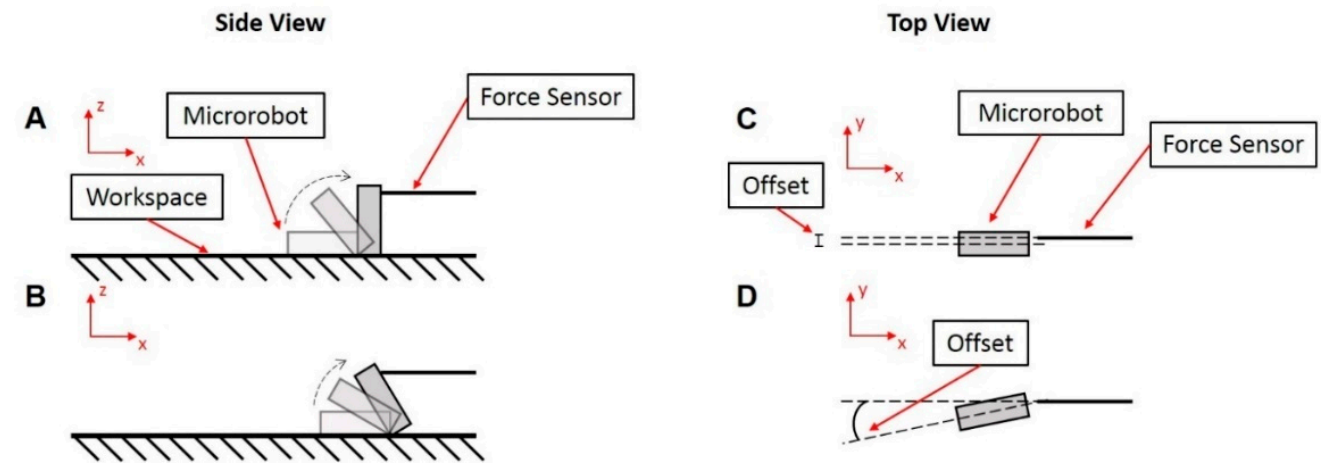

Figure 7. Schematic of various contact scenarios that result in different force measurement readings. These positions/offsets are not mutually exclusive and can occur in combination with each other. (A) Ideal position to directly contact the force sensor resulting in a maximum force reading. (B-D) Positioning offsets resulting in lower force readings: (B) Position offset in the $x$ direction. (C) Position offset in the $y$ direction. (D) Angle offset in the $x y$ plane.

Table 4. Microrobot actuation force. All static tests were conducted using the same conditions, whereas the dynamic tests were conducted at both 1.0 and $1.5 \mathrm{~Hz}$. For the static case, each test corresponds to four different readings, followed by a manual realignment of the microrobot. For the dynamic case, the microrobot is realigned after each force reading (tests).

\begin{tabular}{cccccccccc}
\hline & \multicolumn{4}{c}{ Static Forces $(\mu \mathbf{N})$} & \multicolumn{5}{c}{ Dynamic Forces $(\mu \mathbf{N})$} \\
\hline & Trial 1 & Trial 2 & Trial 3 & Trial 4 & Test 1 & Test 2 & Test 3 & Test 4 \\
Test 1 & 31.94 & 8.89 & 5.95 & 3.76 & $1 \mathrm{~Hz}$ & 2.85 & 6.71 & 5.15 & 9.94 \\
Test 2 & 28.98 & 6.29 & 4.51 & 3.23 & $1.5 \mathrm{~Hz}$ & 13.23 & 2.07 & 7.24 & 10.60 \\
Test 3 & 42.10 & 6.19 & 4.21 & 4.84 & & & & & \\
\hline
\end{tabular}

\section{Discussion}

A microrobot system that is capable of actuating and imaging a tumbling magnetic robot in various biomedical environments, including that of a live murine specimen was demonstrated. When seeded with murine fibroblasts, all material variants of the microrobot exhibited cell proliferation, with no statistically significant difference in toxicity compared to the negative control samples. High-frequency ultrasound imaging allowed for the real-time determination of the microrobot's location in the presence of tissue occlusion. Based on velocity data recorded from the ex vivo porcine test case, the SU-8 lengthwise tumbling variant of the microrobot was determined to have the highest average translation speed and thus used for all subsequent tests. PDMS is much more flexible than rigid SU-8 and tends to conform to minute surface variations under load. Additionally, the resultant surface finish on the PDMS microrobots is much stickier than that of SU-8 microrobots, which are smoother and glossy. We suspect that SU-8 microrobots are faster than the PDMS due to loose fecal matter sticking to the surface of the PDMS microrobots after they are inserted into fluid-filled colon. This outer coating of additional particles would reduce the PDMS microrobots' contact area with the substrate and increase 
slipping occurrences, leading to slower velocities. The SU-8 microrobot variant was shown to diffuse the majority of a fluorescein payload gradually over a 1-h time period and shown to be incapable of puncturing or harming tissue through magnetic force alone.

The design of the microrobot has qualities that are well-posed for in vivo biomedical applications. Magnetic fields harmlessly penetrate living tissue with little to no attenuation or distortion. Clinical usage of magnetic resonance imaging (MRI) machines is widespread and static fields less than 8 Tesla (T) in strength are safe for human use [45]. Magnetic field strength rapidly decreases over distance, compromising mobility if a microrobot is too far from the field source. This scenario is likely to occur in minimally invasive operations, where the target location is far from the point of entry. Therefore, improved magnetic response and stronger field sources are advantageous. To this end, the tumbling magnetic microrobot incorporates neodymium particles and uses actuation based on magnetic torque. Compared to other common magnetic materials such as nickel or ferrite, neodymium exhibits higher remanent magnetization and stronger resistance to demagnetization. Torque-based actuation is generally preferred at the microscale due to its higher efficiency compared to magnetic gradient-based actuation [46]. At further distances with lower magnetic field strengths, sideways tumbling variants can be used to keep the microrobot system operational. These sideways tumbling microrobots require less torque to rotate than their lengthwise tumbling counterparts, due to their smaller moment arm and rotational inertia, but at the cost of lower translational speeds [37]. Tumbling magnetic locomotion, regardless of orientation, was shown to be versatile over the complex and unstructured porcine/murine terrains tested.

The actuating magnetic field itself can be supplied using electromagnetic coils or permanent magnets, but the latter option was incorporated into our system due to space constraints and its simplicity of implementation. Permanent magnets have been used for actuation in a variety of other robotic systems, including helical microrobot platforms [47-49], a microrobotic system for aligning floating electronic circuits to fibers in a wet transfer process [50], and a larger system involving magnetic capsule endoscopes [51]. More recently, point-to-point closed-loop motion control of magnetically driven screws actuated using permanent magnets was demonstrated within an agar gel tissue phantom [52]. For further improving system capabilities, attaching permanent magnet-based systems to robotic arms can increase the available degrees of freedom [53] and incorporating two synchronized rotating magnets can mitigate the detrimental attractive forces exerted on microrobots by rotating single dipoles [48,49]. Additionally, a permanent magnetic system designed for steering catheters demonstrated that such systems are scalable and capable of delivering $80 \mathrm{mT}$ fields over the space of a human torso [54].

A limitation of the tumbling microrobot system is that the microrobot is constrained to $2 \mathrm{D}$ movement on the surface of the environment. Due to its rigid body and uniform magnetic alignment, the microrobot lacks other locomotive modalities outside of tumbling. In addition, the single actuating permanent magnet is unable to produce spatially complex and time-varying magnetic fields. The field strength is static and field gradients cannot be decoupled from the field orientation. Spatial 3D movement is possible for helical magnetic microswimmers under rotational fields [55], but these are restricted to usage in wet environments only. Hu et al. developed an elastomeric neodymium millirobot with nonuniform magnetic alignment capable of multimodal locomotive gaits [56]. By precisely controlling the external magnetic field strength, orientation, and gradient, the millirobot could be coerced into jumping, crawling, swimming, tumbling, and walking gaits. The fabrication of similar soft-bodied robots at the microscale and the improvement of the magnetic field manipulator's capabilities is currently being explored.

The high density of their embedded magnetic particles allows the microrobots to be visualized through ultrasound imaging. Differences in the resultant acoustic impedance between the microrobots and the fluid environment make them distinguishable from their surroundings. Higher image resolution can be achieved by increasing the frequency of the ultrasound waves, at the cost of reduced penetration depth. Because the microrobots must stay within the boundaries of the ultrasound 
beam width and slice thickness to be imaged, out-of-plane motion is not possible without physical manipulation of the ultrasound transducer. It must be relocated in coordination with the microrobots in order to keep them in sight. The use of volumetric 4D ultrasound imaging may relax this requirement, but such technologies cannot yet operate in real time. Thus, all ultrasound imaging in this study had the microrobots tumbling along the scanning plane of the ultrasound transducer.

For the in vivo tests, presented here, we have focused on determining the ideal rotating frequency for the permanent magnet that provides adequate speed for locomotion in the colon along with ease in ultrasound imaging. There is a direct relationship between the distance between the permanent magnet and the force it can apply to the microrobot in the workspace. In our future work with larger animal models, the magnetic field strength needed for locomotion can thus be easily modified using a stronger permanent magnet and/or tuned by adjusting its offset distance from the workspace.

Despite these limitations, in vivo locomotion and imaging of the tumbling microrobot within murine/porcine colons was successfully demonstrated, suggesting the potential use of microrobots towards future clinical applications. There were sensitivity and technical challenges associated with imaging the microrobots in a murine colon largely due to the small size of the animal. This difficulty will likely be diminished in larger animals and humans where a larger colon has the potential to make manipulating the microrobots easier. Colonoscopies, which are necessary to examine and diagnose colorectal cancer and inflammatory bowel disease, are of particular interest. Due to the invasiveness of the conventional colonoscopy procedure, patients often experience extreme discomfort and reluctance to undergo further examination [57]. Furthermore, colonoscopies themselves can exacerbate existing disease symptoms [58]. The use of ultra-thin colonoscopes has been shown to significantly improve tolerability in patients and noninvasive options such as bowel ultrasounds and quantitative fecal immunochemical tests are also available for partial screening, but no solution has fully eliminated the need for colonoscopies [59-61]. The introduction of a microrobotic alternative, however, could lead to new noninvasive procedures that reduce patient discomfort and open new possibilities in disease diagnosis. Additionally, patients with inflammatory bowel disease (IBD) suffer from chronic inflammation, which is a major risk factor for developing gastrointestinal tumors [62-64]. Furthermore, patients with IBD have also been shown to have increased risks of developing extraintestinal malignancies and colorectal cancer, principally resulting from the effects of chronic intestinal inflammation [65]. Therapies that reduce the mucosal inflammatory response are the standard of treatment for IBD. However, the current treatment of inflammatory bowel disease is suboptimal despite the array of available pharmacotherapeutics. These systemic therapies have limited efficacy as well as significant short- and long-term risks for serious adverse effects. A powerful, targeted therapy that could be delivered locally with reasonable precision would represent a major breakthrough for this disease. Such a therapy could be targeted to those specific areas where inflammation, tissue edema, and ulceration are present, allowing for efficient and effective treatment of the affected area without treatment being "wasted" on intact areas of the colon, and of course, decreasing or eliminating the need for systemic therapies. Therefore, this is also a very promising potential clinical application for the microrobots.

The tumbling microrobot, with its in vivo locomotion capabilities and medical imaging visibility, is a viable platform for a host of biomedical applications. The payload coating and diffusion experiments presented in this paper were focused on identifying a feasible technique to apply a payload to the microrobot and the ability of such a payload to passively diffuse from the microrobot. In the future, this generic payload can be replaced with an actual drug payload and applied in a similar manner. The drug payload itself can be coated with a polymer or other material with a characterized diffusion profile to allow for the timed release of the therapeutic agent once the microrobot is at the desired in vivo location. This tumbling microrobot can also be used as a platform for advanced microsensor technologies that could eventually be used to measure microbiota levels, other biological entities in the environment, or as tiny end-effectors capable of performing in vivo microsurgical tasks, including minimally invasive 
biopsies. Furthermore, this platform can be used in the future to target other organs, which have similar fluid content and/or limited accessibility, such as the stomach, intestines, and even the human brain.

In conclusion, a microrobot system capable of real-time manipulation and imaging in in vitro, in situ, ex vivo, and in vivo environments was presented. The system's tumbling microrobot was shown to be viable to cells when compared to the negative control and exerted forces within safe ranges. A mock fluorescein payload demonstrated potential functionalization for targeted drug delivery. Although locomotion tests were primarily conducted within the colon, the microrobot system may prove to be valuable for in vivo biomedical applications in other areas of the body.

Supplementary Materials: The following are available online at http://www.mdpi.com/2072-666X/11/9/861/s1, calculation of the external field magnetic flux density, the estimation of the maximum force exerted on the microrobot, and an overview of the motorized permanent magnet manipulator, Figure S1: Fabrication process, Figure S2: Electrospraying, Figure S3: SEM images of microrobot, Figure S4: Force testing, Figure S5: Cell proliferation, Figure S6: Motorized permanent magnet manipulator, Figure S7: ex vivo tests, Figure S8: Ex vivo velocities, Figure S9: In situ dissected tests, Figure S10: In situ intact tests, Table S1: Magnetic flux density, Movie S1: Ultrasound imaging of porcine locomotion, Movie S2: Ultrasound imaging of murine-agarose locomotion.

Author Contributions: E.E.N., C.B., G.A., E.L., L.S., C.J.G., and D.J.C. conceived of and designed the experiments; G.A. and C.B. performed robot fabrication; C.B. developed the magnetic control system model, designed and fabricated the experimental testbed, and prepared the supplemental video; E.E.N. performed experiments with ultrasound imaging and murine models; E.L. performed cytotoxicity and drug release studies; G.A. performed force testing experiments; E.E.N., C.B., G.A., E.L., L.S., C.J.G., and D.J.C. analyzed the data; E.E.N. and C.B. prepared the original draft paper; E.E.N., C.B., G.A., E.L., L.S., C.J.G., and D.J.C. reviewed and edited the draft; E.E.N. and C.B. prepared the supplemental material. All authors have read and agreed to the published version of the manuscript.

Funding: This work was supported by NSF IIS Award \#1358446 and NSF NRI Award \#1637961. E.E.N. was supported by a Mark and Pamela Lamp Graduate Scholarship. L.S. and E.L. were supported by the National Cancer Institute (R0CA19829 to L.S.).

Acknowledgments: The authors acknowledge the facility access at the Birck Nanotechnology Center at the Purdue University.

Conflicts of Interest: The authors declare no conflict of interest.

\section{References}

1. Luo, M.; Feng, Y.; Wang, T.; Guan, J. Micro-/Nanorobots at Work in Active Drug Delivery. Adv. Funct. Mater. 2018, 28. [CrossRef]

2. Erkoc, P.; Yasa, I.C.; Ceylan, H.; Yasa, O.; Alapan, Y.; Sitti, M.; Yasa, O.; Sitti, M.; Erkoc, P.; Yasa, I.C.; et al. Mobile Microrobots for Active Therapeutic Delivery. Adv. Ther. 2018, 2, 1800064. [CrossRef]

3. Min, J.; Yang, Y.; Wu, Z.; Gao, W. Robotics in the Gut. Adv. Ther. 2019, 3, 1900125. [CrossRef]

4. Kagan, D.; Benchimol, M.J.; Claussen, J.C.; Chuluun-Erdene, E.; Esener, S.; Wang, J. Acoustic droplet vaporization and propulsion of perfluorocarbon-loaded microbullets for targeted tissue penetration and deformation. Angew. Chem. Int. Ed. 2012, 51, 7519-7522. [CrossRef] [PubMed]

5. Ceylan, H.; Yasa, I.C.; Yasa, O.; Tabak, A.F.; Giltinan, J.; Sitti, M. 3D-Printed Biodegradable Microswimmer for Theranostic Cargo Delivery and Release. ACS Nano 2019, 13, 3353-3362. [CrossRef] [PubMed]

6. Wang, X.; Li, J.; Kawazoe, N.; Chen, G. Photothermal ablation of cancer cells by albumin-modified gold nanorods and activation of dendritic cells. Materials 2018, 12, 31. [CrossRef]

7. He, L.; Liu, J.; Li, S.; Feng, X.; Wang, C.; Zhuang, X.; Ding, J.; Chen, X. Polymer Nanoplatforms at Work in Prostate Cancer Therapy. Adv. Ther. 2019, 2, 1800122. [CrossRef]

8. Xu, H.; Medina-Sánchez, M.; Magdanz, V.; Schwarz, L.; Hebenstreit, F.; Schmidt, O.G. Sperm-Hybrid Micromotor for Targeted Drug Delivery. ACS Nano 2018, 12, 327-337. [CrossRef]

9. Mhanna, R.; Qiu, F.; Zhang, L.; Ding, Y.; Sugihara, K.; Zenobi-Wong, M.; Nelson, B.J. Artificial bacterial flagella for remote-controlled targeted single-cell drug delivery. Small 2014, 10, 1953-1957. [CrossRef]

10. Xie, S.; Zhao, L.; Song, X.; Tang, M.; Mo, C.; Li, X. Doxorubicin-conjugated Escherichia coli Nissle 1917 swimmers to achieve tumor targeting and responsive drug release. J. Control. Release 2017, 268, 390-399. [CrossRef] 
11. Li, J.; Li, X.; Luo, T.; Wang, R.; Liu, C.; Chen, S.; Li, D.; Yue, J.; Cheng, S.H.; Sun, D. Development of a magnetic microrobot for carrying and delivering targeted cells. Sci. Robot. 2018, 3, eaat8829. [CrossRef]

12. Qiu, F.; Fujita, S.; Mhanna, R.; Zhang, L.; Simona, B.R.; Nelson, B.J. Magnetic Helical Microswimmers Functionalized with Lipoplexes for Targeted Gene Delivery. Adv. Funct. Mater. 2015, 25, 1666-1671. [CrossRef]

13. Esteban-Fernández de Ávila, B.; Angell, C.; Soto, F.; Lopez-Ramirez, M.A.; Báez, D.F.; Xie, S.; Wang, J.; Chen, Y. Acoustically Propelled Nanomotors for Intracellular siRNA Delivery. ACS Nano 2016, 10, 4997-5005. [CrossRef] [PubMed]

14. Hansen-Bruhn, M.; de Ávila, B.E.F.; Beltrán-Gastélum, M.; Zhao, J.; Ramírez-Herrera, D.E.; Angsantikul, P.; Vesterager Gothelf, K.; Zhang, L.; Wang, J. Active Intracellular Delivery of a Cas9/sgRNA Complex Using Ultrasound-Propelled Nanomotors. Angew. Chem. Int. Ed. 2018, 57, 2657-2661. [CrossRef]

15. Gao, C.; Lin, Z.; Lin, X.; He, Q. Cell Membrane-Camouflaged Colloid Motors for Biomedical Applications. Adv. Ther. 2018, 1, 1800056. [CrossRef]

16. Alford, A.; Rich, M.; Kozlovskaya, V.; Chen, J.; Sherwood, J.; Bolding, M.; Warram, J.; Bao, Y.; Kharlampieva, E. Ultrasound-Triggered Delivery of Anticancer Therapeutics from MRI-Visible Multilayer Microcapsules. Adv. Ther. 2018, 1, 1800051. [CrossRef]

17. Gao, W.; Dong, R.; Thamphiwatana, S.; Li, J.; Gao, W.; Zhang, L.; Wang, J. Artificial micromotors in the mouse's stomach: A step toward in vivo use of synthetic motors. ACS Nano 2015, 9, 117-123. [CrossRef]

18. Leong, T.G.; Randall, C.L.; Benson, B.R.; Bassik, N.; Stern, G.M.; Gracias, D.H. Tetherless thermobiochemically actuated microgrippers. Proc. Natl. Acad. Sci. USA 2009, 106, 703-708. [CrossRef]

19. Gultepe, E.; Randhawa, J.S.; Kadam, S.; Yamanaka, S.; Selaru, F.M.; Shin, E.J.; Kalloo, A.N.; Gracias, D.H. Biopsy with thermally-responsive untethered microtools. Adv. Mater. 2013, 25, 514-519. [CrossRef]

20. de Ávila, B.E.F.; Zhao, M.; Campuzano, S.; Ricci, F.; Pingarrón, J.M.; Mascini, M.; Wang, J. Rapid micromotor-based naked-eye immunoassay. Talanta 2017, 167, 651-657. [CrossRef]

21. Wu, Z.; Li, T.; Gao, W.; Xu, T.; Jurado-Sánchez, B.; Li, J.; Gao, W.; He, Q.; Zhang, L.; Wang, J. Cell-Membrane-Coated Synthetic Nanomotors for Effective Biodetoxification. Adv. Funct. Mater. 2015, 25, 3881-3887. [CrossRef]

22. Zhang, Y.; Yan, K.; Ji, F.; Zhang, L. Enhanced Removal of Toxic Heavy Metals Using Swarming Biohybrid Adsorbents. Adv. Funct. Mater. 2018, 28, 1806340. [CrossRef]

23. Park, S.J.; Park, S.H.; Cho, S.; Kim, D.M.; Lee, Y.; Ko, S.Y.; Hong, Y.; Choy, H.E.; Min, J.J.; Park, J.O.; et al. New paradigm for tumor theranostic methodology using bacteria-based microrobot. Sci. Rep. 2013, 3, 3394. [CrossRef] [PubMed]

24. Wu, Z.; Wu, Y.; He, W.; Lin, X.; Sun, J.; He, Q. Self-propelled polymer-based multilayer nanorockets for transportation and drug release. Angew. Chem. Int. Ed. 2013, 52, 7000-7003. [CrossRef] [PubMed]

25. Servant, A.; Qiu, F.; Mazza, M.; Kostarelos, K.; Nelson, B.J. Controlled in vivo swimming of a swarm of bacteria-like microrobotic flagella. Adv. Mater. 2015, 27, 2981-2988. [CrossRef]

26. Vilela, D.; Cossío, U.; Parmar, J.; Martínez-Villacorta, A.M.; Gómez-Vallejo, V.; Llop, J.; Sánchez, S. Medical Imaging for the Tracking of Micromotors. ACS Nano 2018, 12, 1220-1227. [CrossRef]

27. Jeon, S.; Kim, S.; Ha, S.; Lee, S.; Kim, E.; Kim, S.Y.; Park, S.H.; Jeon, J.H.; Kim, S.W.; Moon, C.; et al. Magnetically actuated microrobots as a platform for stem cell transplantation. Sci. Robot. 2019, 4, 1-12. [CrossRef]

28. Nguyen, P.B.; Park, J.O.; Park, S.; Ko, S.Y. Medical micro-robot navigation using image processing-blood vessel extraction and X-ray calibration. In Proceedings of the IEEE RAS and EMBS International Conference on Biomedical Robotics and Biomechatronics, Singapore, 26-29 June 2016; IEEE Computer Society: Washington, DC, USA, 2016; Volume 2016, pp. 365-370.

29. Nguyen, P.B.; Kang, B.; Bappy, D.M.; Choi, E.; Park, S.; Ko, S.Y.; Park, J.O.; Kim, C.S. Real-time microrobot posture recognition via biplane $\mathrm{X}$-ray imaging system for external electromagnetic actuation. Int. J. Comput. Assist. Radiol. Surg. 2018, 13, 1843-1852. [CrossRef]

30. Sánchez, A.; Magdanz, V.; Schmidt, O.G.; Misra, S. Magnetic control of self-propelled microjets under ultrasound image guidance. In Proceedings of the IEEE RAS and EMBS International Conference on Biomedical Robotics and Biomechatronics, Sao Paulo, Brazil, 12-15 August 2014; IEEE Computer Society: Washington, DC, USA, 2014; pp. 169-174. 
31. Khalil, I.S.M.; Ferreira, P.; Eleutério, R.; De Korte, C.L.; Misra, S. Magnetic-based closed-loop control of paramagnetic microparticles using ultrasound feedback. In Proceedings of the IEEE International Conference on Robotics and Automation, Hong Kong, China, 31 May-7 June 2014; Institute of Electrical and Electronics Engineers Inc.: Piscataway, NJ, USA, 2014; pp. 3807-3812.

32. Rahmer, J.; Stehning, C.; Gleich, B. Remote magnetic actuation using a clinical scale system. PLoS ONE 2018, 13. [CrossRef]

33. Yan, X.; Zhou, Q.; Vincent, M.; Deng, Y.; Yu, J.; Xu, J.; Xu, T.; Tang, T.; Bian, L.; Wang, Y.X.J.; et al. Multifunctional biohybrid magnetite microrobots for imaging-guided therapy. Sci. Robot. 2017, 2, eaaq1155. [CrossRef]

34. Wang, B.; Zhang, Y.; Zhang, L. Recent progress on micro- and nano-robots: Towards in vivo tracking and localization. Quant. Imaging Med. Surg. 2018, 8, 461-479. [CrossRef] [PubMed]

35. Bi, C.; Niedert, E.E.; Adam, G.; Lambert, E.; Solorio, L.; Goergen, C.J.; Cappelleri, D.J. Tumbling Magnetic Microrobots for Biomedical Applications. In Proceedings of the MARSS 2019: The 4th International Conference on Manipulation, Automation and Robotics at Small Scales, Helsinki, Finland, 1-5 July 2019.

36. Freeling, J.L.; Rezvani, K. Assessment of murine colorectal cancer by micro-ultrasound using three dimensional reconstruction and non-linear contrast imaging. Mol. Ther. Methods Clin. Dev. 2016, 3, 16070. [CrossRef] [PubMed]

37. Bi, C.; Guix, M.; Johnson, B.V.; Jing, W.; Cappelleri, D.J. Design of microscale magnetic tumbling robots for locomotion in multiple environments and complex terrains. Micromachines 2018, 9, 68. [CrossRef]

38. Camacho, J.M.; Sosa, V. Alternative method to calculate the magnetic field of permanent magnets with azimuthal symmetry. Rev. Mex. Física E 2013, 59, 8-17.

39. Alin, A.; Kurt, S. Testing non-additivity (interaction) in two-way ANOVA tables with no replication. Stat. Methods Med. Res. 2006, 15, 63-85. [CrossRef]

40. Driscoll, W.C. Robustness of the ANOVA and Tukey-Kramer statistical tests. Comput. Ind. Eng. 1996, 31, 265-268. [CrossRef]

41. Tylose for Personal Care; SE Tylose GmbH \& Co.: Wiesbaden, Germany, 2015. Available online: https: //www.essentialingredients.com/pdf/tyloseforpersonalcare.pdf (accessed on 17 September 2020).

42. 510(k) Summary of Safety and Effectveness [21 CFR 807.92(c)]. 2014. Available online: https://www. accessdata.fda.gov/scripts/cdrh/cfdocs/cfPMN/pmn.cfm?ID=K130581 (accessed on 17 September 2020).

43. Ozbek, H. Viscosity of Aqueous Sodium Chloride Solutions from 0-150 ${ }^{\circ} \mathrm{C}$; Lawrence Berkeley National Lab. (LBNL): Berkeley, CA, USA, 2010.

44. Bao, X.; Li, W.; Lu, M.; Zhou, Z.R. Experiment study on puncture force between MIS suture needle and soft tissue. Biosurf. Biotribol. 2016, 2, 49-58. [CrossRef]

45. Schenck, J.F. Safety of strong, static magnetic fields. J. Magn. Reson. Imaging 2000, 12, 2-19. [CrossRef]

46. Ceylan, H.; Giltinan, J.; Kozielski, K.; Sitti, M. Mobile microrobots for bioengineering applications. Lab Chip 2017, 17, 1705-1724. [CrossRef]

47. Fountain, T.W.R.; Kailat, P.V.; Abbott, J.J. Wireless Control of Magnetic Helical Microrobots using a Rotating-Permanent-Magnet Manipulator. In Proceedings of the IEEE International Conference on Robotics and Automation, Anchorage, AK, USA, 3-8 May 2010.

48. Alshafeei, M.E.; Hosney, A.; Klingner, A.; Misra, S.; Khalil, I.S.M. Magnetic-Based Motion Control of a Helical Robot using Two Synchronized Rotating Dipole Fields. In Proceedings of the 5th IEEE RAS \& EMBS International Conference on Biomedical Robotics and Biomechatronics (BioRob), São Paulo, Brazil, 12-15 August 2014.

49. Hosney, A.; Klingner, A.; Misra, S.; Khalil, I.S.M. Propulsion and steering of helical magnetic microrobots using two synchronized rotating dipole fields in three-dimensional space. In Proceedings of the IEEE International Conference on Intelligent Robots and Systems, Hamburg, Germany, 28 September-2 October 2015; Institute of Electrical and Electronics Engineers Inc.: Hamburg, Germany, 2015; Volume 2015, pp. 1988-1993.

50. Barbot, A.; Tan, H.; Power, M.; Seichepine, F.; Yang, G.-Z. Floating magnetic microrobots for fiber functionalization. Sci. Robot. 2019, 4, eaax8336. [CrossRef]

51. Mahoney, A.W.; Abbott, J.J. Five-degree-of-freedom manipulation of an untethered magnetic device in fluid using a single permanent magnet with application in stomach capsule endoscopy. Int. J. Rob. Res. 2016, 35, 129-147. [CrossRef] 
52. Zhang, Z.; Klingner, A.; Misra, S.; Khalil, I.S.M. Control of Magnetically-Driven Screws in a Viscoelastic Medium. In Proceedings of the IEEE/RSJ International Conference on Intelligent Robots and Systems (IROS), Las Vegas, NV, USA, 25-29 October 2020.

53. Mahoney, A.W.; Abbott, J.J. Generating Rotating Magnetic Fields With a Single Permanent Magnet for Propulsion of Untethered Magnetic Devices in a Lumen. IEEE Trans. Robot. 2014, 30, 411. [CrossRef]

54. Tsuchida, K.; García-García, H.M.; van der Giessen, W.J.; McFadden, E.P.; van der Ent, M.; Sianos, G.; Meulenbrug, H.; Ong, A.T.L.; Serruys, P.W. Guidewire navigation in coronary artery stenoses using a novel magnetic navigation system: First clinical experience. Catheter. Cardiovasc. Interv. 2006, 67, 356-363. [CrossRef] [PubMed]

55. Abbott, J.J.; Peyer, K.E.; Lagomarsino, M.C.; Zhang, L.; Dong, L.; Kaliakatsos, I.K.; Nelson, B.J. How Should Microrobots Swim? Int. J. Rob. Res. 2009, 28, 1434-1447. [CrossRef]

56. Hu, W.; Lum, G.Z.; Mastrangeli, M.; Sitti, M. Small-scale soft-bodied robot with multimodal locomotion. Nature 2018, 554, 81-85. [CrossRef]

57. Denters, M.J.; Schreuder, M.; Depla, A.C.; Mallant-Hent, R.C.; van Kouwen, M.C.A.; Deutekom, M.; Bossuyt, P.M.; Fockens, P.; Dekker, E. Patients' perception of colonoscopy: Patients with inflammatory bowel disease and irritable bowel syndrome experience the largest burden. Eur. J. Gastroenterol. Hepatol. 2013, 25, 964-972. [CrossRef]

58. Menees, S.; Higgins, P.; Korsnes, S.; Elta, G. Does colonoscopy cause increased ulcerative colitis symptoms? Inflamm. Bowel Dis. 2007, 13, 12-18. [CrossRef]

59. Ogawa, T.; Ohda, Y.; Nagase, K.; Kono, T.; Tozawa, K.; Tomita, T.; Iimuro, M.; Hida, N.; Oshima, T.; Fukui, H.; et al. Evaluation of discomfort during colonoscopy with conventional and ultrathin colonoscopes in ulcerative colitis patients. Dig. Endosc. 2015, 27, 99-105. [CrossRef]

60. Parente, F.; Molteni, M.; Marino, B.; Colli, A.; Ardizzone, S.; Greco, S.; Sampietro, G.; Foschi, D.; Gallus, S. Are colonoscopy and bowel ultrasound useful for assessing response to short-term therapy and predicting disease outcome of moderate-to-severe forms of ulcerative colitis: A prospective study. Am. J. Gastroenterol. 2010, 105, 1150-1157. [CrossRef]

61. Takashima, S.; Kato, J.; Hiraoka, S.; Nakarai, A.; Takei, D.; Inokuchi, T.; Sugihara, Y.; Takahara, M.; Harada, K.; Okada, H.; et al. Evaluation of mucosal healing in ulcerative colitis by fecal calprotectin vs. fecal immunochemical test. Am. J. Gastroenterol. 2015, 110, 873-880. [CrossRef]

62. Choi, C.H.R.; Rutter, M.D.; Askari, A.; Lee, G.H.; Warusavitarne, J.; Moorghen, M.; Thomas-Gibson, S.; Saunders, B.P.; Graham, T.A.; Hart, A.L. Forty-year analysis of colonoscopic surveillance program for neoplasia in ulcerative colitis: An updated overview. Am. J. Gastroenterol. 2015, 110, 1022-1034. [CrossRef] [PubMed]

63. Eaden, J.A.; Abrams, K.R.; Mayberry, J.F. The risk of colorectal cancer in ulcerative colitis: A meta-analysis. Gut 2001, 48, 526-535. [CrossRef] [PubMed]

64. Ullman, T.A.; Itzkowitz, S.H. Intestinal inflammation and cancer. Gastroenterology 2011, 140, 1807-1816.e1. [CrossRef]

65. Axelrad, J.E.; Lichtiger, S.; Yajnik, V. Inflammatory bowel disease and cancer: The role of inflammation, immunosuppression, and cancer treatment. World J. Gastroenterol. 2016, 22, 4794-4801. [CrossRef] [PubMed]

(C) 2020 by the authors. Licensee MDPI, Basel, Switzerland. This article is an open access article distributed under the terms and conditions of the Creative Commons Attribution (CC BY) license (http://creativecommons.org/licenses/by/4.0/). 